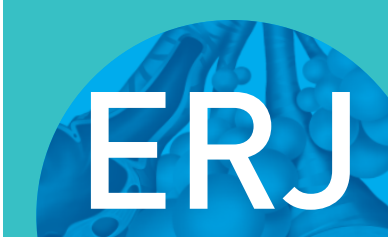

open research

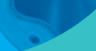

\section{Dexmedetomidine sedation for endobronchial ultrasound-guided transbronchial needle aspiration, a randomised controlled trial}

\author{
Ting-Yu Lin $0^{1}$, Yu-Chen Huang ${ }^{1}$, Chih-Hsi Kuo ${ }^{1}$, Fu-Tsai Chung ${ }^{1}$, Yu-Ting Lin², \\ Tsai-Yu Wang (10) ${ }^{1}$, Shu-Min Lin (10) ${ }^{1}$ and Yu-Lun Lo ${ }^{1}$
}

Affiliations: 'Dept of Thoracic Medicine, Chang Gung Memorial Hospital, Chang Gung University, College of Medicine, Taipei, Taiwan. ${ }^{2}$ Dept of Anesthesiology, Taipei-Veterans General Hospital, Taipei, Taiwan.

Correspondence: Yu-Lun Lo, Dept of Thoracic Medicine, Chang Gung Memorial Hospital, 199 Tun-Hwa N. Rd, Taipei, Taiwan. E-mail: loyulundahotmail.com

\section{ABSTRACT}

Background and aim: Appropriate sedation is important to the success of endobronchial ultrasound-guided transbronchial needle aspiration (EBUS-TBNA). Dexmedetomidine is a sedative agent that operates via the $\alpha 2$ adrenergic agonist, which provides sleep-like sedation with little respiratory suppression. This study compared the efficacy and safety of dexmedetomidine sedation with propofol in cases of EBUS-TBNA.

Methods: Patients requiring EBUS-TBNA were randomly assigned dexmedetomidine sedation $(D, n=25)$ or propofol sedation $(\mathrm{P}, \mathrm{n}=25)$. Vital signs, diagnostic yield and the bispectral index (BIS) were recorded throughout the bronchoscopic procedure and recovery period. The tolerance and cooperation of the patients were evaluated using questionnaires.

Measurements and results: The lowest mean arterial blood pressure in group D (79.2 \pm 9.9 versus $72.5 \pm 12.9 \mathrm{mmHg}, \mathrm{p}=0.049)$ exceeded that in group $\mathrm{P}$, the lowest heart rate was lower $(60.9 \pm 10.2$ versus $71.4 \pm 11.8$ beats $\left.\cdot \mathrm{min}^{-1}, \mathrm{p}=0.006\right)$ and the mean BIS during sedation was significantly higher $(84.1 \pm 8.3$ versus $73.6 \pm 5.7, \mathrm{p}<0.001)$. Patients in group $\mathrm{D}$ were more likely to report perceiving procedure-related symptoms and express an unwillingness to undergo the bronchoscopy again, if indicated (41.1 versus $83.3 \%, \mathrm{p}=0.007$ ). One subject in group D aborted EBUS-TBNA due to intolerance. Many of the variables in the two groups were similar, including the proportion of hypoxaemic events, recovery times, patient cooperation and diagnostic yield.

Conclusions: The effects of dexmedetomidine on haemodynamics were in line with its pharmacodynamic features. Patients who received dexmedetomidine were more likely than those who received propofol to perceive the procedures. Overall, dexmedetomidine did not prove inferior to propofol sedation in terms of patient cooperation or diagnostic yield.

@ERSpublications

Compared to propofol sedation for EBUS-TBNA, dexmedetomidine provided patients lighter sedation with lower heart rates and less decrease in blood pressure. The recovery times, hypoxaemia, cooperation and diagnostic yield in the two groups were similar. https://bit.ly/33qgEj3

Cite this article as: Lin T-Y, Huang Y-C, Kuo $\mathrm{C}-\mathrm{H}$, et al. Dexmedetomidine sedation for endobronchial ultrasound-guided transbronchial needle aspiration, a randomised controlled trial. ERJ Open Res 2020; 6: 00064-2020 [https://doi.org/10.1183/23120541.00064-2020].

This article has supplementary material available from openres.ersjournals.com

This study is registered at www.clinicaltrials.gov with identifier number NCT03521505. Individual participant data that underline the results reported in this article will be shared, after deidentification (text, tables, figures and appendix).

Received: 11 Feb 2020 | Accepted after revision: 4 Sept 2020

Copyright $\odot$ ERS 2020. This article is open access and distributed under the terms of the Creative Commons Attribution Non-Commercial Licence 4.0 . 


\section{Introduction}

Endobronchial ultrasound (EBUS)-guided transbronchial needle aspiration (TBNA) is an essential procedure for lung cancer diagnosis and staging. Sampling mediastinal lymph nodes using EBUS-TBNA is far easier than conventional mediastinoscopy [1]. Note that this procedure is performed orally, and multiple sampling is required. Note also that performing additional procedures, such as endobronchial or transbronchial lung biopsy, can greatly prolong the duration of the procedure. Under these conditions, appropriate sedation is essential to ensuring patient cooperation and minimising patient discomfort throughout the entire procedure [2-4].

Propofol sedation is ideally suited to flexible bronchoscopy, due to its rapid onset and short-term effects [5-7]. Propofol acts mainly through $\gamma$-aminobutyric acid (GABA) receptor potentiation. It tends to decrease the central respiratory drive as well as pharyngeal muscle tone [8-11]. Physicians overseeing propofol sedation should undergo training specifically for this drug. Target-controlled infusion and bispectral index (BIS) monitors are commonly used to prevent cardiopulmonary depression due to oversedation [12-16]. Despite a growing body of evidence supporting the efficacy and safety of propofol, the use of this drug for procedural sedation by non-anaesthesiologists is limited in many countries [17]. Midazolam is the standard alternative to propofol sedation; however, further options, such as dexmedetomidine, should also be explored.

Dexmedetomidine has been approved in Europe and the United States for its sedative and analgesic effects. The effects are exerted via $\alpha 2$ adrenergic receptors in the locus coeruleus and dorsal horn of the spinal cord. The fact that dexmedetomidine has relatively little effect on muscles of the upper airway greatly limits respiratory depression during sedation [18-22]. Among the cardiovascular effects is a decrease in the heart rate and blood pressure within hours of infusion [23]. Dexmedetomidine induces sleep-like sedation, from which the patient is easily aroused. The patients also tend to be more cooperative and display better cognitive function [24, 25].

Our aim in this study was to compare the effectiveness of dexmedetomidine and propofol for EBUS-TBNA in terms of cardiopulmonary parameters, patient tolerance, cooperation and diagnostic yield. For those areas where pulmonologists cannot perform propofol sedation (for example due to legislative requirements) the current study aims to explore alternatives for sedation in EBUS-TBNA if propofol cannot be used. Because many studies have compared midazolam to propofol, we decided to investigate dexmedetomidine, which pulmonologists use for sedation of patients on mechanical ventilation in current practice. Furthermore, no previous study has shown EBUS-TBNA diagnostic yield and detailed sedative profiles among patients receiving dexmedetomidine. Therefore, we conducted this prospective study to provide pulmonologists with scientific evidence of the role of dexmedetomidine in EBUS-TBNA sedation. Some of the results have been published previously in abstracts from the ERS International Congress 2019 [26].

\section{Methods}

This prospective open-label randomised study was conducted in a medical centre (Chang Gung Memorial Hospital, Linkou, Taiwan). The study protocol was approved by the Chang Gung Medical Foundation Institutional Review Board (No.201601093A3). The trial was registered at clinicaltrials.gov (NCT03521505). Patients who required EBUS-TBNA and agreed to undergo the procedure under sedation were screened for enrolment. The exclusion criteria included age $<20$ years, American Society of Anaesthesiologists (ASA) physical status classification 4 or 5 , a Mallampati score of 4 , severe sleep apnoea syndrome (apnoea-hypopnea index $>40$ ), second or third degree atrioventricular blockage, heart rate $<50$ beats per minute, systolic blood pressure $<90 \mathrm{mmHg}$, neurological disorders or other conditions contributing to difficulty in assessing response, body mass index $>42$ in males or $>35$ in females, and pregnancy. Patients with a known history of allergy to the study drugs or to eggs, soybeans or sulfite products were also excluded. All enrolled patients provided written informed consent. Enrolled patients were randomised into the study group or the control group using a predetermined random computer code at a ratio of 1:1.

\section{Patient preparation}

Blood pressure was monitored using an automated pressure cuff, and heart rate (HR) was monitored using a three-lead ECG. A peripheral pulse oximeter was used to monitor oxygen saturation measured by pulse oximetry $\left(S_{\mathrm{pO}_{2}}\right)$, while a nasal cannula delivered oxygen at a rate of $2 \mathrm{~L} \cdot \mathrm{min}^{-1}$. A disposable BIS Quatro Sensor (Aspect Medical System Inc, Newton, MA, USA) was applied to the forehead of patients. Smoothening time was set at $15 \mathrm{~s}$ [12]. The BIS level was covered (i.e., blinded to the investigator in charge of sedation). A patient monitor (Philips MP60, Bobling, Germany) was used to continuously record all parameters except for the blood pressure, which was recorded every $2.5 \mathrm{~min}$. The monitoring software was developed in Microsoft Visual Basic 6.0 (Windows XP) based on the Philip Patient Monitor communication protocol. An intravenous catheter was placed in the forearm for drug administration. An 
oral bite block was placed prior to sedation. Premedication was achieved using nebulised $2 \%$ xylocaine inhalation.

The investigators in charge of sedation were specifically trained in the administration of sedatives and monitoring sedative depth $[12,13,15,27-29]$. In our hospital, physicians who administer sedation must receive training on how to monitor the sedative depth, the pharmacology of sedative drugs and the risk assessments required for sedation. The training is overseen by anaesthesiologists, and participants must take and pass a test every 2 years. The investigators in charge of sedation were responsible for monitoring patients for cardiopulmonary depression and determining the need for interventions. The interventions are detailed in the supplementary material. EBUS-TBNA operations were performed by experienced bronchoscopists (Kuo C-H and Chung F-T) using a convex probe endobronchial ultrasound (BF-UC260FW, Olympus, Tokyo, Japan) via the oral route, with assistance from a well-trained technician.

\section{Sedation protocol}

Study group: Alfentanil $\left(5 \mu \mathrm{g} \cdot \mathrm{kg}^{-1}\right)$ was administered to patients in a 1:10 dilution with normal saline under slow injection for $2 \mathrm{~min}$ prior to full induction using an infusion of dexmedetomidine $\left(1 \mu \mathrm{g} \cdot \mathrm{kg}^{-1}\right)$ for $10 \mathrm{~min}[18,22,30]$. Maintenance was conducted via dexmedetomidine infusion $\left(0.5-1.4 \mu \mathrm{g} \cdot \mathrm{kg}^{-1} \cdot \mathrm{h}^{-1}\right)$ with the aim of maintaining stable vital signs and Observer Assessment of Alertness and Sedation scale (OAA/S) score of 2-3.

Control group: Alfentanil $\left(5 \mu \mathrm{g} \cdot \mathrm{kg}^{-1}\right)$ was slowly administered to patients in a 1:10 dilution with normal saline for $2 \mathrm{~min}$ prior to induction employing a propofol infusion at an initial effect-site concentration (Ce) of $2.0 \mu \mathrm{g} \cdot \mathrm{mL}^{-1}$ using an Injectomat ${ }^{\mathrm{R}}$ TIVA Agilia (Fresenius Kabi, France) [11, 13]. OAA/S was evaluated every $30 \mathrm{~s}$ after the patients closed their eyes. In cases where OAA/S did not reach 3 when Ce reached $2.0 \mu \mathrm{g} \cdot \mathrm{mL}^{-1}$, Ce was increased by $0.2 \mu \mathrm{g} \cdot \mathrm{mL}^{-1}$ every $90 \mathrm{~s}$ until the OAA/S score reached $2-3$. For maintenance of the control group, Ce of propofol was titrated at a rate of $0.2 \mu \mathrm{g} \cdot \mathrm{mL}^{-1}$ every $90 \mathrm{~s}$ to achieve stable vital signs and an OAA/S score of 2-3.

Following the procedure, the patients were monitored continuously in a recovery room until they had fully recovered.

\section{Assessment}

$\mathrm{S}_{\mathrm{pO}_{2}}$, blood pressure, $\mathrm{HR}$ and BIS were recorded immediately before induction (as a baseline), during induction, during the maintenance of sedation and throughout the recovery period. The parameter levels and the difference from baseline values were analysed. We recorded episodes of hypoxaemia $\left(S_{\mathrm{pO}_{2}}<90 \%\right)$ and hypotension (mean arterial blood pressure (MAP) $<65 \mathrm{mmHg}$ or systolic blood pressure (SBP) $<90 \mathrm{mmHg}$ ) of any duration. Sedative drug doses were recorded at the infusion pump. Procedure time was recorded as the duration from the insertion of bronchoscope to its removal. Recovery time was recorded as the duration between the time at which bronchoscopy finished and the time when the patients spontaneously opened their eyes and were able to recall their date of birth and correctly perform the finger-to-nose test.

After recovery, patients were asked to answer a questionnaire about wakefulness, tolerance and willingness to repeat the bronchoscopic procedure if clinically indicated. Wakefulness during the procedure was evaluated by asking patients whether they heard or saw anything during the operation. The questionnaire used to assess patient tolerance to procedure-related symptoms included reactions to nebulised xylocaine inhalation, stimulation caused by insertion of the scope through the mouth, cough, dyspnoea, pain and global discomfort to the entire procedure. The questionnaire used a $100-\mathrm{mm}$ visual analogue scale (VAS) (0: no bother, 100: intolerable). Patients were also asked about their willingness to undergo the bronchoscopic procedure again if clinically indicated (definitely not, possibly not, not sure, possibly yes and definitely yes). The bronchoscopist was questioned concerning the ease of scope insertion and biopsy, coughing by the patient and global cooperation throughout the procedure using a $100-\mathrm{mm}$ VAS (0: most cooperative, 100: entirely uncooperative).

The diagnostic yield of EBUS-TBNA was evaluated in terms of the pathology or cytology of mediastinal lymph nodes. Specimens without lymphocytes were defined as inadequate samples. There are two criteria by which to confirm a result as a true negative. The first is confirming a lack of malignancy in specimens obtained surgically. The second is a confirmation of stability or regression via computed tomography at 6 months after the procedure [31]. 
dexmedetomidine sedation and $36.7 \%$ of those under propofol sedation experienced episodes of hypoxaemia $[13,18,22]$. Considering the complexity of EBUS-TBNA, a difference of $30 \%$ would be of clinical importance $(\alpha=0.05$, power $=0.8)$. Our analysis revealed that 25 subjects per group would be sufficient to detect a difference between the two groups.

\section{Statistical analysis}

Analysis was performed on the outcomes of all randomised subjects. The diagnostic yield among subjects who completed EBUS-TBNA was expressed as a number with a percentage or a mean with standard deviation. Continuous variables were evaluated using the Mann-Whitney test. Patient characteristics and complications were analysed using the Chi-squared test or Fisher's exact test in cases where the sample size was small. A $\mathrm{p}<0.05$ was considered statistically significant. All statistical analysis was performed using Prism 5 (GraphPad software Inc., San Diego, CA, USA).

\section{Results}

The trial was conducted from May 2018 through January 2020. Five subjects who declined to join the study during the screening phase were excluded. The remaining 50 subjects were randomly assigned dexmedetomidine or propofol for sedation. Both groups presented comparable patient characteristics and indications, and the add-on procedures in the two groups were similar (table 1). The main add-on procedures were mini-probe endobronchial ultrasound and transbronchial lung biopsy. Baseline blood pressure, $\mathrm{HR}, \mathrm{S}_{\mathrm{pO}_{2}}$ and BIS levels were comparable in the two groups (supplementary table S1). One subject who underwent dexmedetomidine sedation was unable to complete EBUS-TBNA due to intolerance.

During induction (supplementary table S2), the incidence of hypoxaemia and hypotension was similar in the two groups. HR in the dexmedetomidine group was significantly lower than in the propofol group. BIS levels in the propofol group were lower than in the dexmedetomidine group.

During maintenance (table 2), blood pressure and BIS levels in the dexmedetomidine group were significantly higher than in the propofol group, whereas HRs were lower. The incidence of hypoxaemia was similar in the two groups. All of the patients that presented with hypoxaemia or hypotension recovered spontaneously under proper management. There were no occurrences of intubation, mortality or severe bleeding. During the recovery period, the effects of sedative drugs on vital signs vanished in both groups; however, HR in the dexmedetomidine group remained lower than in the propofol group (supplementary table S3).

\begin{tabular}{|c|c|c|c|}
\hline & Dexmedetomidine & Propofol & p-value \\
\hline Subjects $\mathrm{n}$ & 25 & 25 & \\
\hline \multicolumn{4}{|l|}{ Patient characteristics } \\
\hline Age years & $59.7 \pm 14.1$ & $59.4 \pm 12.2$ & 0.9 \\
\hline ASA & $3(1-3)$ & $3(1-3)$ & 0.7 \\
\hline Male & $14(56.0)$ & $13(52.0)$ & 1.0 \\
\hline Body mass index $\mathrm{kg} \cdot \mathrm{m}^{-2}$ & $23.6 \pm 3.9$ & $23.6 \pm 3.8$ & 0.8 \\
\hline Mallampati score & $2(1-3)$ & $2(1-3)$ & 0.5 \\
\hline Intolerance and withdrawal & $1(7.0)$ & 0 & 1.0 \\
\hline Procedure time ${ }^{\#} \min$ & $30.0 \pm 13.2$ & $27.6 \pm 8.6$ & 0.7 \\
\hline \multicolumn{4}{|l|}{ Add-on procedures } \\
\hline Miniprobe endobronchial ultrasound & $16(64.0)$ & $12(48.0)$ & 0.4 \\
\hline Transbronchial lung biopsy & $12(48.0)$ & $11(44.0)$ & 1.0 \\
\hline Bronchial wash & $13(52.0)$ & $11(44.0)$ & 0.8 \\
\hline Bronchoalveolar lavage & $3(12.0)$ & $4(16.0)$ & 1.0 \\
\hline Total doses of sedatives $\mathrm{mg}$ & $95.6 \pm 29.5$ & $184.1 \pm 64.4$ & \\
\hline Recovery time ${ }^{\pi} \min$ & $12.4 \pm 11.1$ & $6.2 \pm 4.8$ & 0.06 \\
\hline
\end{tabular}

Data are presented as mean \pm SD, $n(\%)$ or median (range), unless otherwise stated. ASA: American Society of Anaesthesiologists physical status classification. ${ }^{\#}$ : duration from the insertion of bronchoscope to its removal; ": duration between the time of finishing bronchoscopic procedure and the time when the patients could spontaneously open their eyes, recall their date of birth and correctly perform the finger-to-nose test. 


\begin{tabular}{|c|c|c|c|}
\hline Events & Dexmedetomidine & Propofol & p-value \\
\hline Subjects $\mathrm{n}$ & 25 & 25 & \\
\hline \multicolumn{4}{|l|}{ Blood pressure $\mathrm{mmHg}$} \\
\hline Lowest MAP & $79.2 \pm 9.9$ & $72.5 \pm 12.9$ & 0.04 \\
\hline$\triangle M A P^{\|}$ & $-16.7 \pm 13.8$ & $-21.9 \pm 13.6$ & 0.1 \\
\hline MAP $<65 \mathrm{mmHg}$ & $1(4.0)$ & $5(20.0)$ & 0.2 \\
\hline Lowest SBP & $106.7 \pm 13.8$ & $98.2 \pm 13.4$ & 0.049 \\
\hline$\triangle \mathrm{SBP}^{\Uparrow}$ & $-24.9 \pm 15.7$ & $-36.0 \pm 23.2$ & 0.1 \\
\hline $\mathrm{SBP}<90 \mathrm{mmHg}$ & $3(12.0)$ & $7(28.0)$ & 0.3 \\
\hline \multicolumn{4}{|l|}{ Heart rate beats. $\min ^{-1}$} \\
\hline Lowest heart rate & $60.9 \pm 10.2$ & $71.4 \pm 11.8$ & 0.006 \\
\hline$\Delta$ heart rate & $-13.6 \pm 9.3$ & $-3.3 \pm 9.1$ & $<0.001$ \\
\hline Heart rate $<60$ beats $\cdot \mathrm{min}^{-1}$ & $12(48.0)$ & $4(16.0)$ & 0.03 \\
\hline \multicolumn{4}{|l|}{ Oxygenation \% } \\
\hline Lowest $S_{\mathrm{pO}_{2}}$ & $90.9 \pm 5.2$ & $87.9 \pm 6.3$ & 0.1 \\
\hline$\Delta S_{\mathrm{pO}_{2}}{ }^{\mathrm{l}}$ & $-8.3 \pm 5.3$ & $-10.9 \pm 6.2$ & 0.1 \\
\hline $\mathrm{S}_{\mathrm{pO}_{2}}<90 \%$ & 8 (32.0) & $14(56.0)$ & 0.2 \\
\hline Mean BIS & $84.1 \pm 8.3$ & $73.6 \pm 5.7$ & $<0.001$ \\
\hline
\end{tabular}

Following recovery, one subject in each of the groups refused to answer the questionnaire, such that 48 patients were evaluated for wakefulness during bronchoscopy, patient tolerance and willingness to undergo the procedure again. Patients in the dexmedetomidine group were more likely to report wakefulness than those in the propofol group (figure 1). Patients in the dexmedetomidine group were also more likely to report hearing something $(58.3 \%$ versus $16.7 \%, \mathrm{p}=0.007)$ or seeing something $(20.8 \%$ versus $4.2 \%, \mathrm{p}=0.2)$ while under sedation. In terms of tolerance, patients in the dexmedetomidine group were more likely to perceive procedure-related symptoms, as indicated by VAS values (figure 2a). The proportion of patients that unreservedly agreed to repeated bronchoscopy (if indicated) was higher in the propofol group than in the dexmedetomidine group ( $41.1 \%$ versus $83.3 \%, \mathrm{p}=0.007$, figure 3 ). The attending bronchoscopists reported that patient cooperation and ease of biopsy during bronchoscope insertion as well as coughing by the patient were similar in the two groups.

Samples adequate for pathological examination were obtained from patients in both groups: dexmedetomidine group ( 29 nodes) and propofol group (32 nodes). Three nodes from one patient in the propofol group were excluded from analysis because the patient was lost from follow-up, such that negative nodal biopsy results could not be judged as a true negative (table 3). The diagnostic yield was comparable in the two groups in terms of true positive rate $(48.3 \%$ versus $37.9 \%)$, true negative rate $(48.3 \%$ versus $48.3 \%)$ and false negative rate (3.4\% versus $13.8 \%)$.

FIGURE 1 Wakefulness during sedation. Following recovery, patients were asked if they had seen or heard anything during the bronchoscopic procedure. One subject in each group refused to answer the questionnaire.

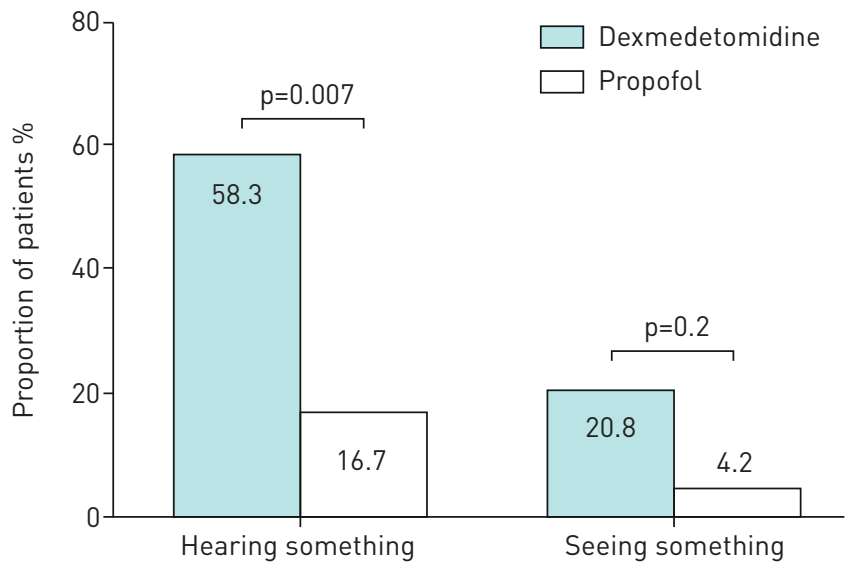



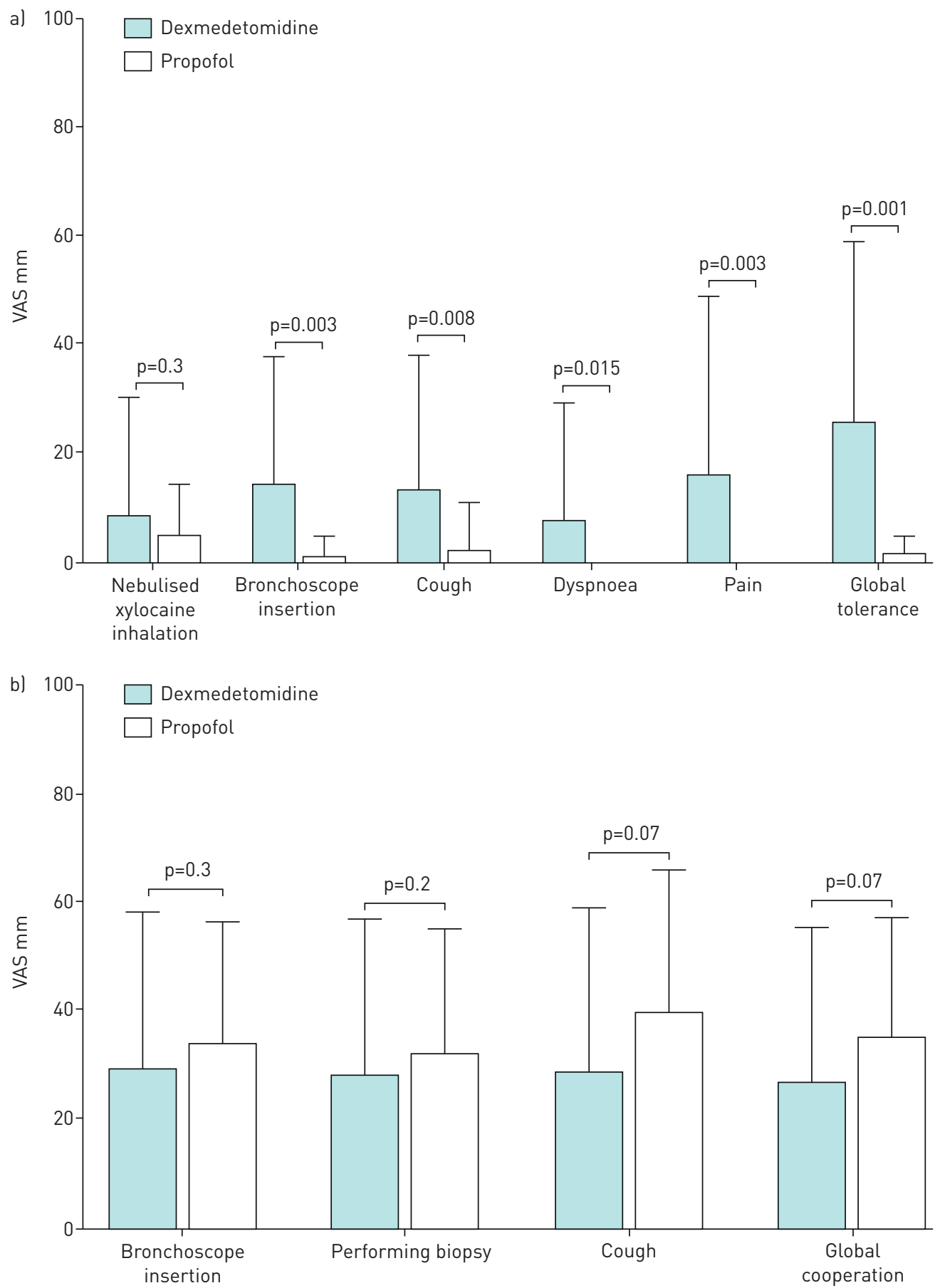

FIGURE 2 a) Patient tolerance for procedure-related symptoms. Following recovery, subjects answered a questionnaire on procedure-related symptoms, including reactions to nebulised xylocaine inhalation, stimulation caused by scope insertion through the mouth, coughing, dyspnoea, pain and global tolerance to the entire procedure. Note that one subject in each group refused to answer the questionnaire. b) Patient cooperation during the bronchoscopic procedure. Bronchoscopist answered a questionnaire on the ease of scope insertion and biopsy, coughing by the patient and global cooperation during the procedure. The design of the questionnaire was based on a 100-mm visual analogue scale (VAS, 0: no bother, 100: worst intolerable/uncooperative).

\section{Discussion}

Our results revealed that compared to patients that underwent propofol sedation for EBUS-TBNA, those who underwent dexmedetomidine sedation experienced lighter sedation with lower HRs and a less pronounced decrease in blood pressure. Note that recovery times and the incidence of hypoxaemia in the two groups were similar. No severe complications were encountered in either group. Patients in the 


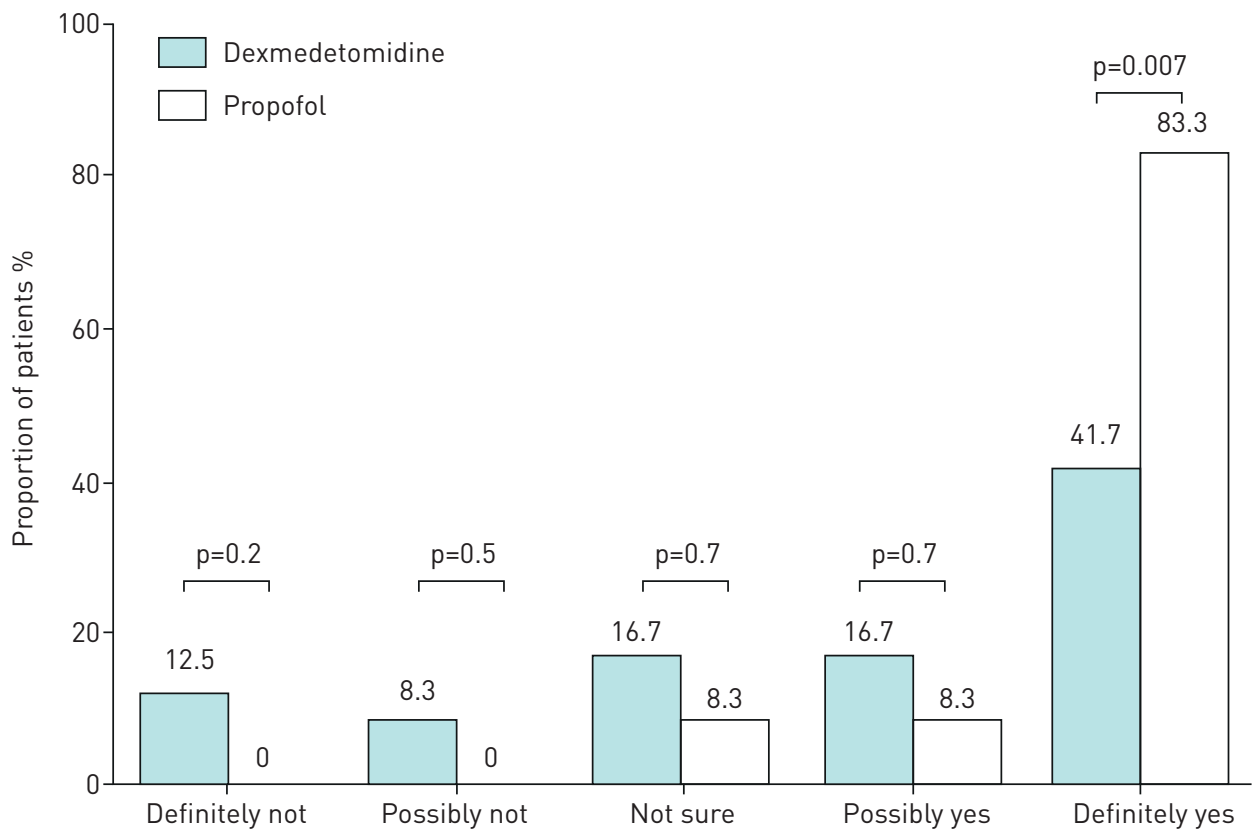

FIGURE 3 Willingness to undergo repeated bronchoscopic procedure. Following recovery, patients were queried about their willingness to undergo the procedure again if indicated clinically (definitely not, possibly not, not sure, possibly yes and definitely yes). One subject in each group refused to answer the questionnaire.

dexmedetomidine group were more likely to perceive procedure-related symptoms and were less likely to express a willingness to repeat the procedure if indicated. Patients in both groups displayed similar degrees of cooperation during EBUS-TBNA and similar diagnostic yield.

The beneficial effects of dexmedetomidine in terms of respiratory depression did not transfer to oxygenation outcomes in the present study. This may be due to the availability of supplemental $\mathrm{O}_{2}$ from a nasal cannula for safety. This also implies that the factors contributing to hypoxaemia during EBUS-TBNA are multifactorial, such that they could not be improved by a single drug. It appears that

TABLE 3 Diagnostic yield of endobronchial ultrasound-guided transbronchial needle aspiration in the two groups

Dexmedetomidine

$$
\begin{aligned}
& 7 \\
& 4 \\
& 2 \\
& 10 \\
& 11
\end{aligned}
$$$$
\text { Lymph nodes } \mathrm{n}
$$$$
\text { Lymph node station }
$$

Adequate nodal number for pathology Diagnostic yield

True positive

Malignancy

Sarcoidosis

True negative

False negative

Malignancy

Sarcoidosis

Thyroid nodal hyperplasia
29

11

8

1

5

4

29

14 (48.3)

13

1

14 (48.3)

1 (3.4)

1

0

0
Propofol

32

13

11

1

5

2

32

11 (37.9)

8

$2,1=3^{\#}$

14 (48.3)

$4(13.8)$

2

1

1

Data are presented as $\mathrm{n}$ or $\mathrm{n}(\%)$, unless otherwise stated. ${ }^{\#}$ : the three nodes from one patient in the propofol group were excluded from analysis of diagnostic yield because the patient was lost from follow-up, such that negative nodal biopsy results could not be judged as a true negative. 
procedure-related secretion, coughing, individual cardiopulmonary capacity and drug metabolisation are also possible factors. The study results confirmed our expectations based on the pharmacokinetic features of the drugs that HR in the dexmedetomidine group would be lower than in the propofol group and blood pressure would be higher. Li et al. [32] reported the clinical efficacy of a combination of dexmedetomidine and propofol for general anaesthesia and compared it with propofol alone for bronchoscopy. The stress index and required propofol doses were less in the group receiving the combination of dexmedetomidine and propofol. Like the present study, the intraoperative $\mathrm{HR}$ was lower in the patients receiving dexmedetomidine. This study demonstrated the advantage of combining dexmedetomidine in bronchoscopic sedation. However, a laryngeal mask was required to maintain adequate ventilation during general anaesthesia. Cases of severe bradycardia and cardiac arrest have previously been linked to dexmedetomidine sedation when used for other procedures or for general anaesthesia [33, 34]. In the current study, the mean HR of patients who received dexmedetomidine was $18 \%$ below the baseline during maintenance and $13 \%$ lower during recovery; however, none of the patients required further intervention to correct this (table 2 and supplementary table S2). Patients presenting a risk of bradycardia were excluded during screening, and sedation was limited to the dose at which the patient achieved the desired sedative level. Full recovery was confirmed before the patients left the room in which the bronchoscopic procedure was performed.

Based on the BIS levels, we determined that the sedation induced by dexmedetomidine was lighter than that induced by propofol. This difference could be attributed to the properties of the drugs as well as the sedation protocol. Lighter sedation may explain why patients in the dexmedetomidine group were more likely to perceive the procedure and were less likely to express a willingness to undergo the procedure again. Nonetheless, the less pronounced amnesia effects of dexmedetomidine should be taken into consideration when dealing with patients who are subject to high anxiety. RADEK et al. [35] also showed patients receiving dexmedetomidine had greater awareness than patients receiving propofol, which is consistent with our finding. Thus, it is crucial that physicians explain the contingencies of EBUS-TBNA under sedation. Note that the bronchoscopists assigned similar grades for patient cooperation and diagnostic yield in the two groups. One patient who received dexmedetomidine was unable to tolerate the procedure. Note that dexmedetomidine is not indicated for patients experiencing profound anxiety such that an alternative regimen should be administered. Further studies should be conducted to determine the feasibility of using a low dose of midazolam as premedication prior to the administration of dexmedetomidine [36]. Based on the results obtained in this study, we will undertake a pharmacodynamic study on the use of dexmedetomidine titration to improve amnesia effects and overcome the effects of suppressed cardiopulmonary function.

Most previous studies on the use of sedation for EBUS-TBNA focused on the diagnostic yield. All but one retrospective study reported that diagnostic yield and patient tolerance under deep propofol sedation was equivalent to that under moderate midazolam sedation [37-40]. Prospective randomised controlled trials comparing the use of propofol for general anaesthesia and midazolam for moderate sedation were also comparable in terms of diagnostic yield, major complications and patient tolerance [31, 41]. In the current study, we compared the diagnostic yield and detailed sedative profiles among patients undergoing dexmedetomidine or propofol sedation from the perspective of patients, bronchoscopists and physicians overseeing sedation. The information obtained in this study could help to improve patient selection for EBUS-TBNA under sedation.

The present study has a number of limitations. First, we did not exclude cases where procedures other than EBUS-TBNA were performed, as was done in other prospective trials [31, 41]. Thus, this study encountered many situations that arise in real-world practice. Note also that the add-on procedures and procedure duration were equally distributed in the two groups. Second, the investigators in charge of sedation were not blinded to the patients' titration regimen. Nonetheless, the safety profiles of the sedative drugs were in line with their pharmacodynamic features. Furthermore, the patients reported their own feelings related to the effects of sedation, which is important to real-life practice. Third, because the number of subjects was small, a large-scale study is needed to confirm the results of the present study.

Compared to propofol sedation for EBUS-TBNA, dexmedetomidine resulted in lighter sedation, a more pronounced reduction in $\mathrm{HR}$ and less pronounced reduction in blood pressure. The incidence of hypoxaemia and the time required for recovery were similar in the two groups. Overall, dexmedetomidine did not prove inferior to propofol sedation in terms of patient cooperation or diagnostic yield.

Conflict of interest: None declared.

Support statement: This study is supported by the Chang Gung Memorial Hospital (Grant no. CMRPG3F2161, CMRPG3F2162). Funding information for this article has been deposited with the Crossref Funder Registry. 


\section{References}

1 Silvestri GA, Gonzalez AV, Jantz MA, et al. Methods for staging non-small cell lung cancer: Diagnosis and management of lung cancer, 3rd ed: American College of Chest Physicians evidence-based clinical practice guidelines. Chest 2013; 143: Suppl. 5, e211S-e250S.

2 Canneto B, Ferraroli G, Falezza G, et al. Ideal conditions to perform EBUS-TBNA. J Thorac Dis 2017; 9: Suppl. 5, S414-S417.

3 Aswanetmanee $\mathrm{P}$, Limsuwat $\mathrm{C}$, Kabach $\mathrm{M}$, et al. The role of sedation in endobronchial ultrasound-guided transbronchial needle aspiration: systematic review. Endosc Ultrasound 2016; 5: 300-306.

4 Wahidi MM, Sterman DH. Bringing comfort to endobronchial ultrasound bronchoscopy. Am J Respir Crit Care Med 2015; 191: 727-728.

5 Stolz D, Kurer G, Meyer A, et al. Propofol versus combined sedation in flexible bronchoscopy: a randomised non-inferiority trial. Eur Respir J 2009; 34: 1024-1030.

6 Clarkson K, Power CK, O'Connell F, et al. A comparative evaluation of propofol and midazolam as sedative agents in fiberoptic bronchoscopy. Chest 1993; 104: 1029-1031

7 Crawford M, Pollock J, Anderson K, et al. Comparison of midazolam with propofol for sedation in outpatient bronchoscopy. Br J Anaesth 1993; 70: 419-422.

8 Murphy M, Bruno MA, Riedner BA, et al. Propofol anesthesia and sleep: a high-density EEG study. Sleep 2011; 34: $283-291 \mathrm{~A}$

9 Hillman DR, Walsh JH, Maddison KJ, et al. Evolution of changes in upper airway collapsibility during slow induction of anesthesia with propofol. Anesthesiology 2009; 111: 63-71.

10 Eastwood PR, Platt PR, Shepherd K, et al. Collapsibility of the upper airway at different concentrations of propofol anesthesia. Anesthesiology 2005; 103: 470-477.

11 Lin T-Y, Fang Y-F, Huang S-H, et al. Capnography monitoring the hypoventilation during the induction of bronchoscopic sedation: a randomized controlled trial. Sci Rep 2017; 7: 8685.

12 Lo YL, Lin TY, Fang YF, et al. Feasibility of bispectral index-guided propofol infusion for flexible bronchoscopy sedation: a randomized controlled trial. PLoS One 2011; 6: e27769.

13 Lin TY, Lo YL, Hsieh CH, et al. The potential regimen of target-controlled infusion of propofol in flexible bronchoscopy sedation: a randomized controlled trial. PLoS One 2013; 8: e62744.

14 Clark G, Licker M, Younossian AB, et al. Titrated sedation with propofol or midazolam for flexible bronchoscopy: a randomised trial. Eur Respir J 2009; 34: 1277-1283

15 American Society of Anesthesiologists Task Force on Sedation and Analgesia by Non-Anesthesiologists. Practice guidelines for sedation and analgesia by non-anesthesiologists. Anesthesiology 2002; 96: 1004-1017.

16 Wahidi MM, Jain P, Jantz M, et al. American College of Chest Physicians consensus statement on the use of topical anesthesia, analgesia, and sedation during flexible bronchoscopy in adult patients. Chest 2011; 140: 1342-1350.

17 Perel A. Non-anaesthesiologists should not be allowed to administer propofol for procedural sedation: a Consensus Statement of 21 European National Societies of Anaesthesia. Eur J Anaesthesiol 2011; 28: 580-584.

18 Yuan F, Fu H, Yang P, et al. Dexmedetomidine-fentanyl versus propofol-fentanyl in flexible bronchoscopy: a randomized study. Exp Ther Med 2016; 12: 506-512.

19 Goneppanavar U, Magazine R, Periyadka Janardhana B, et al. Intravenous dexmedetomidine provides superior patient comfort and tolerance compared to intravenous midazolam in patients undergoing flexible bronchoscopy. Pulm Med 2015; 2015: 727530.

20 Ehsan Z, Mahmoud M, Shott SR, et al. The effects of anesthesia and opioids on the upper airway: a systematic review. Laryngoscope 2016; 126: 270-284.

21 Venn RM, Hell J, Grounds RM. Respiratory effects of dexmedetomidine in the surgical patient requiring intensive care. Crit Care 2000; 4: 302-308.

22 Ryu JH, Lee SW, Lee JH, et al. Randomized double-blind study of remifentanil and dexmedetomidine for flexible bronchoscopy. Br J Anaesth 2012; 108: 503-511.

23 Shehabi Y, Ruettimann U, Adamson $\mathrm{H}$, et al. Dexmedetomidine infusion for more than 24 hours in critically ill patients: sedative and cardiovascular effects. Intensive Care Med 2004; 30: 2188-2196.

24 Venn RM, Grounds RM. Comparison between dexmedetomidine and propofol for sedation in the intensive care unit: patient and clinician perceptions. Br J Anaesth 2001; 87: 684-690.

25 Nelson LE, Lu J, Guo T, et al. The alpha2-adrenoceptor agonist dexmedetomidine converges on an endogenous sleep-promoting pathway to exert its sedative effects. Anesthesiology 2003; 98: 428-436.

26 Lin T-Y, Kuo C-H, Chung F-T, et al. Comparison of dexmedetomidine to propofol for sedation of endobronchial ultrasound-guided transbronchial needle aspiration. Eur Respir J 2019; 54: Suppl. 63, PA320.

27 Jose RJ, Shaefi S, Navani N. Sedation for flexible bronchoscopy: current and emerging evidence. Eur Respir Rev 2013; 22: 106-116.

28 Ni YL, Lo YL, Lin TY, et al. Conscious sedation reduces patient discomfort and improves satisfaction in flexible bronchoscopy. Chang Gung Med J 2010; 33: 443-452.

29 Fanning RM. Monitoring during sedation given by non-anaesthetic doctors. Anaesthesia 2008; 63: 370-374.

30 Conti G, Ranieri VM, Costa R, et al. Effects of dexmedetomidine and propofol on patient-ventilator interaction in difficult-to-wean, mechanically ventilated patients: a prospective, open-label, randomised, multicentre study. Cri Care 2016; 20: 206.

31 Casal RF, Lazarus DR, Kuhl K, et al. Randomized trial of endobronchial ultrasound-guided transbronchial needle aspiration under general anesthesia versus moderate sedation. Am J Respir Crit Care Med 2015; 191: 796-803.

$32 \mathrm{Li} \mathrm{H}$, Zhang $\mathrm{N}$, Zhang $\mathrm{K}$, et al. Observation of the clinical efficacy of dexmedetomidine in flexible bronchoscopy under general anesthesia: clinical case experience exchange. J Int Med Res 2019; 47: 6215-6222.

33 Ingersoll-Weng E, Manecke GR, Jr., Thistlethwaite PA. Dexmedetomidine and cardiac arrest. Anesthesiology 2004; 100: 738-739.

34 Gerlach AT, Murphy CV. Dexmedetomidine-associated bradycardia progressing to pulseless electrical activity: case report and review of the literature. Pharmacotherapy 2009; 29: 1492.

35 Radek L, Kallionpaa RE, Karvonen M, et al. Dreaming and awareness during dexmedetomidine- and propofol-induced unresponsiveness. Br J Anaesth 2018; 121: 260-269. 
Houghton CM, Raghuram A, Sullivan PJ, et al. Pre-medication for bronchoscopy: a randomised double blind trial comparing alfentanil with midazolam. Respir Med 2004; 98: 1102-1107.

37 Oztas S, Aka Akturk U, Alpay LA, et al. A comparison of propofol-midazolam and midazolam alone for sedation in endobronchial ultrasound-guided transbronchial needle aspiration: a retrospective cohort study. Clin Respir J 2017; 11: 935-941.

38 Franzen D, Schneiter D, Weder W, et al. Impact of sedation technique on the diagnostic accuracy of endobronchial ultrasound-guided transbronchial needle aspiration. Endosc Ultrasound 2017; 6: 257-263.

39 Conte SC, Spagnol G, Confalonieri M, et al. Deep sedation versus minimal sedation during endobronchial ultrasound transbronchial needle aspiration. Monaldi Arch Chest Dis 2018; 88: 967.

40 Yarmus LB, Akulian JA, Gilbert C, et al. Comparison of moderate versus deep sedation for endobronchial ultrasound transbronchial needle aspiration. Ann Am Thorac Soc 2013; 10: 121-126.

41 Fernandes MGO, Santos VF, Martins N, et al. Endobronchial ultrasound under moderate sedation versus general anesthesia. J Clin Med 2018; 7: 421. 